\title{
BMJ Open Study protocol: a prospective controlled clinical trial to assess surgical or medical treatment for paediatric type 2 diabetes ( $\left.\mathrm{ST}_{2} \mathrm{OMP}\right)$
}

\author{
Amy S Shah (D) , ${ }^{1}$ Michael A Helmrath, ${ }^{2}$ Thomas $\mathrm{H}$ Inge, ${ }^{3}$ Stavra A Xanthakos, ${ }^{4}$ \\ Megan M Kelsey, ${ }^{5}$ Todd Jenkins, ${ }^{2}$ Andrew T Trout, ${ }^{6}$ Lorna Browne, ${ }^{7}$ \\ Kristen J Nadeau ${ }^{3}$
}

To cite: Shah AS, Helmrath MA, Inge TH, et al. Study protocol: a prospective controlled clinical trial to assess surgical or medical treatment for paediatric type 2 diabetes (ST 0 OMP). BMJ Open 2021;11:e047766. doi:10.1136/ bmjopen-2020-047766

- Prepublication history for this paper is available online. To view these files, please visit the journal online (http://dx.doi. org/10.1136/bmjopen-2020047766).

Received 09 December 2020 Accepted 23 July 2021

Check for updates

(c) Author(s) (or their employer(s)) 2021. Re-use permitted under CC BY-NC. No commercial re-use. See rights and permissions. Published by BMJ.

For numbered affiliations see end of article.

Correspondence to

Dr Amy S Shah;

amy.shah@cchmc.org

\section{ABSTRACT}

Introduction The pathophysiology of type 2 diabetes (T2D) in youth differs from adults and conventional medical treatment approaches with lifestyle change, metformin, thiazolidinediones or insulin are inadequate. Metabolic bariatric surgery (MBS) improves multiple health outcomes in adults with T2D. Initial small, uncontrolled studies of Roux-en-Y gastric bypass have also suggested beneficial effects in adolescents. Definitive studies in youth with T2D are lacking, especially with the now more common form of MBS, vertical sleeve gastrectomy (VSG). The surgical or medical treatment for paediatric type 2 diabetes (ST $\mathrm{OMP}_{2}$ clinical trial was designed to test the hypothesis that VSG will more effectively reduce hyperglycaemic and diabetes comorbidities than the best currently available medical treatment incorporating state of the art pharmacotherapies. $\mathrm{ST}_{2} \mathrm{OMP}$ is also designed to better understand the pancreatic and enterohepatic mechanisms by which MBS improves diabetes and its associated comorbidities.

Methods and analysis $\mathrm{ST}_{2} \mathrm{OMP}$ is a prospective, open-label, controlled clinical trial that will recruit 90 postpubertal participants, age range 13-19.9 years, with $\geq 35 \mathrm{~kg} / \mathrm{m}^{2}$ or $>120 \%$ of 95 th percentile and youthonset T2D. The primary outcome is the per cent of youth achieving haemoglobin A1c $<6.0 \%$ at 12 months postgroup allocation (post-VSG vs postmedical group allocation). Secondary outcomes include remission of comorbidities and measures of $\beta$-cell and incretin responses at 12 and 24 months post VSG versus AMT. Ethics and dissemination The $\mathrm{ST}_{2} \mathrm{OMP}$ protocol was approved by the Cincinnati Children's Hospital Medical Center and the University of Colorado Institutional Review Boards. Written informed consent is obtained prior to study enrolment. Study findings will be widely disseminated through peer-reviewed publications and conference presentations.

Trial registration number Clinical Trials.Gov NCT04128995.

\section{INTRODUCTION}

Twenty-seven percent of youth with type 2 diabetes (T2D) in population-based cohorts have poor glycaemic control, defined very
Strengths and limitations of this study

- This is a non-randomised, prospective clinical trial that will compare outcomes in adolescents who self-select advanced medical therapy with vertical sleeve gastrectomy to those who self-select advanced medical therapy alone.

- Advanced medical therapy includes diet and exercise counselling and incorporates the use of multidrug therapy, if needed, to achieve a haemoglobin ( $\mathrm{Hb}) \mathrm{A} 1 \mathrm{c}$ of $<6.5 \%$.

- The primary outcome is the percent of youth achieving $\mathrm{HbA1C}<6.0 \%$ at 12 months postgroup allocation. Secondary outcomes include the impact of treatments on diabetes-related comorbidities, beta cell function and incretin responses.

- Limitations of the study include lack of randomisation of participants and self-selection of their treatment arm.

conservatively as a haemoglobin $(\mathrm{Hb}) \mathrm{A} 1 \mathrm{c}$ $>9.5 \% .{ }^{12}$ In the Treatment Options for Type 2 Diabetes in Adolescents and Youth (TODAY) randomised clinical trial, metformin and metformin plus lifestyle were ineffective in maintaining durable glycaemic control (HbAlc $<8.5 \%$ ) in $57 \%$ and $47 \%$ of adolescents with youth-onset T2D, respectively. ${ }^{3}$ Moreover, in the TODAY study, while rosiglitazone enhanced the efficacy of metformin, the combined dual therapy was still ineffective at maintaining durable control in over one-third of adolescents. ${ }^{3}$ Importantly, both insulin and thiazolidinediones (TZDs) promote weight gain and TZDs have ongoing heart failure risk concerns. Data from the TODAY study also showed high rates of early diabetes complications and continued disease progression over time. ${ }^{4-6}$

The Restoring Insulin Secretion (RISE) clinical trial treated youth with prediabetes or T2D 
with metformin alone or insulin followed by metformin and both regimens were ineffective at improving $\beta$-cell function or slowing glycaemic progression. When youth were compared with adults, adults actually achieved better glycaemic outcomes in the RISE trial. ${ }^{78}$ Recent results from the Ellipse study tested the glucagon-like peptide (GLP-1) agonist liraglutide in 10-17year olds with T2D and found liraglutide lowered the 26-week primary efficacy end point $\mathrm{HbAlc}$ by 0.64 percentage points (vs an increase by 0.42 percentage points with placebo), that is, a treatment difference of -1.06 percentage points $(\mathrm{p}<0.001)$; the difference increased to -1.30 percentage points by 52 weeks, ${ }^{9}$ leading to US Food and Drug Adminstration (FDA) approval of liraglutide for youth-onset T2D. However, GLP-1 analogue studies have not yet looked at longer-term durability of glycaemic control or the impact of the medication on diabetes-related complications. Thus, currently-available paediatric T2D treatment algorithms ${ }^{10}{ }^{11}$ do not achieve glycaemic targets or prevent diabetes progression. Innovative approaches to slow $\beta$-cell decline improve glycaemic control, and durably reduce comorbidities in youth-onset T2D are urgently needed.

Bariatric surgery induces weight loss. However, as efficacy data emerged in adults also showing high rates of T2D, hypertension (HTN) and dyslipidaemia remission as well as reductions in HbAlc, medication use and T2D-related microvascular and macrovascular complications, the term 'metabolic bariatric surgery' (MBS) was coined. ${ }^{12-16}$ MBS now appears to be the most effective T2D treatment in adults. ${ }^{17}$ Up to 27 years of follow-up in the adult Swedish Obese Subject (SOS) trial demonstrated that MBS with a variety of operations induced durable reductions of $25 \%$ in body weight, $30 \%$ in mortality, and $50 \%$ in microvascular complications versus adults not choosing surgery. ${ }^{18}$ Further, the SOS study found a $30 \%$ T2D remission rate with MBS at 15 years versus $6.5 \%$ in the nonsurgical arm. ${ }^{19}$ The Surgical Therapy and Medications Potentially Eradicate Diabetes Efficiently study also reported T2D remission in 23\% of adults 5 years post vertical sleeve gastrectomy (VSG) compared with $5 \%$ with intensive medical treatment. ${ }^{20}{ }^{21}$ Given the numerous differences between youth-onset versus adult-onset T2D including severe insulin resistance, early and rapid $\beta$-cell failure, poor response to lifestyle and medication interventions, and the unique impacts of puberty, growth and adolescent behavior, ${ }^{22}$ it is challenging to extrapolate these adult data to youth.

Teen-Longitudinal Assessment of Bariatric Surgery (LABS), a prospective, observational cohort study of obese youth undergoing bariatric surgery, examined 29 youth with T2D who underwent Riux-en-Y gastric bypass (RYGB). A $94 \%$ (95\% CI $84 \%$ to $100 \%$ ) T2D remission rate occurred at 3 years post-op with no incident T2D cases in the 153 youth without T2D. ${ }^{23}$ When retrospectively comparing these results from the TeenLABS surgical study to outcomes in a matched cohort from the TODAY medical study, youth undergoing RYBG appeared to provide better outcomes than with medical therapy. ${ }^{24}{ }^{25}$ However, neither of these studies were designed for direct comparison with notable differences in study design, outcomes collected and cohort characteristics. In addition, new medical therapies are now available since the timeframe of the TODAY study, arguing the need for a contemporary prospective study. Importantly, almost all the MBS data in youth come from adolescents undergoing RYGB, but there has been a major shift in the type of MBS procedures performed in adolescents to VSG now accounting for $>80 \%$ of adolescent cases. ${ }^{26}{ }^{27} \mathrm{In}$ Teen-LABS, only six youth with T2D underwent VSG; thus very limited outcome data exist for youth with T2D undergoing VSG. In adults, MBS implemented at a younger age, shorter T2D duration $(<5-8$ years), and prior to insulin dependence predicted T2D remission. ${ }^{14}{ }^{28}$ These data provide a strong argument for MBS during adolescence and earlier in the course of T2D.

The current surgical or medical treatment for paediatric type 2 diabetes $\left(\mathrm{ST}_{2} \mathrm{OMP}\right.$ ) trial aims to compare the effect of advanced medical therapy (AMT) plus VSG to AMT alone on glycaemic control and T2D-associated comorbidities. Additionally, the study aims to understand the mechanisms underlying VSG including the impact of VSG on beta cell function and incretin hormones. Our working hypothesis is that AMT plus VSG will be more effective in reducing glycaemic control and T2Dassociated comorbidities than AMT alone, where AMT is defined as aggressive, multidrug, treat-to-target medical therapy aimed at lowering HbA1c.

\section{METHODS AND ANALYSIS}

\section{Study design}

The $\mathrm{ST}_{2} \mathrm{OMP}$ study began its funding period in September 2019. The first patient was enrolled into run-in in Feb 2020. The first baseline study visit was performed in August 2020. The anticipated completion of the study is August 2024.

$\mathrm{ST}_{2} \mathrm{OMP}$ is a prospective, open-label, controlled clinical trial. Randomisation was strongly considered, but was ultimately deemed impossible as: (1) true randomisation is impeded in the USA by inequitable insurance coverage of MBS for youth and surgical costs are too high to be covered by existing grant mechanisms; (2) covering surgical costs would risk potential coercion for some youth to participate to access an otherwise unattainable procedure; and (3) there is potential for high dropout if participants are not randomised to their preferred group. ${ }^{29}$ As shown by others when strong barriers to randomisation exist, as in this study, well-controlled prospective, open-label clinical trials remain a valid and valuable alternatives ${ }^{30} 31$

\section{Recruitment}

Participants are being recruited from the T2D and bariatric surgery clinics at two academic clinical centres, Cincinnati Children's Hospital in Cincinnati, Ohio and Children's Hospital Colorado in Aurora, Colorado. On 
average, each month, both sites participate in the care of $\sim 50$ teens with T2D. The primary recruitment strategy consists of in-person invitations by providers and other members of the multidisciplinary team within the T2D and bariatric surgery clinics. All races and both sexes are recruited. As part of routine clinical care standardised between both sites, all adolescents with T2D are provided lifestyle counselling, and additional therapies for T2D are discussed which include medications, and for those with a body mass index (BMI) $>35$ or $>120 \%$ of 95th percentile, consideration of MBS per the American Society for Metabolic and Bariatric Surgery Pediatric Committee guidelines ${ }^{32}$ and American Academy of Pediatrics policy statement. ${ }^{33}$ As such, all participants have the opportunity to consider MBS. Adolescents self-select to medical or surgical treatment based on personal/ family preference, but is sometimes MBS is declined due to insurance coverage. After adolescents choose their clinical treatment path and if they meet all inclusion and exclusion criteria, they are invited to participate in $\mathrm{ST}_{2} \mathrm{OMP}$. Therefore, treatment arm assignment in $\mathrm{ST}_{2} \mathrm{OMP}$ is self-selected prior to enrolling in the study. Enrolment will continue until both medical and surgical arms of the study achieve their required sample sizes. Written informed consent is obtained from the adolescent if age $\geq 18$ years or the legally authorised representative for youth $<18$ years; for youth $<18$ years, assent is obtained. Any participant who requests withdrawal from the study can do so at any time.

\section{Participants}

This study will recruit 90 participants with youth-onset T2D who are between 13 through $<20$ years of age. T2D is defined as having negative islet cell antibody titers and diabetes onset prior to age 18 years. A BMI $\geq 35 \mathrm{~kg}$ / $\mathrm{m}^{2}$ or $>120$ th of the 95 th percentile based on CDC charts is also required for inclusion at time of enrolment. These BMI criteria were chosen for inclusion because they (1) follow the current best-practice clinical guidelines to consider MBS in adolescents, ${ }^{33}$ (2) allow for BMI-comparable groups at enrolment, and (3) will ensure all participants will have the potential option of surgery, as insurance companies will not presently cover MBS if starting BMI $<35$ or $<120$ th of the 95th percentile. Exclusion criteria for $\mathrm{ST}_{2} \mathrm{OMP}$ include diabetes from causes other than T2D, weight promoting medications within 60 days of enrolment (eg, oral steroids), chronic kidney or liver disease with the exception of non-alcoholic fatty liver disease (NAFLD) or diabetic kidney disease (DKD), pregnancy or breastfeeding, genetic causes of obesity or hypothalamic obesity, history of prior bariatric surgery or history of malignancy. As of April 2021, 75 participants eligible for the study were approached, 22 declined participation and 46 have been enrolled. Of the 46 adolescents enrolled, 15 participants have enrolled in the VSG arm and 31 have enrolled in the AMT arm.

\section{Study design}

Run-In

Participants who meet the study inclusion and exclusion criteria are consented to participate in a 6-month run-in period, regardless of treatment arm. During runin, participants in both the VSG and AMT groups are seen every 3 months by a paediatric endocrinologist per American Diabetes Association (ADA) guidelines ${ }^{10}$ and receive AMT. The definition of AMT includes aggressive multidrug, treat-to-target medical therapy with the goal of achieving an A1c $<6.5 \%$. All visits include nutrition and exercise counselling. Medications are added if A1c is $>6.5 \%$. If Alc is maintained at $<6.5 \%$, medications are continued.

A treat-to-target approach is desirable because it allows flexibility for patient drug preferences, insurance coverage and side effects. ${ }^{11} 34$ Potential medications for use include metformin, basal insulin, GLP-1 agonists and sodium-glucose transport-2 inhibitors. The latter two medication classes have been associated with weight loss and cardiovascular benefit in adults ${ }^{35} 36$ and studies in youth with T2D show adding the GLP-1 agonist liraglutide to metformin in youth with T2D results in a further mean HbA1c lowering of $\sim 0.5 \%-1 \%$ and a favourable safety profile. ${ }^{9}$ Pioglitazone is considered for participants' not tolerating metformin. Insulin is also used short or long term as needed to achieve glycaemia.

The run-in period was designed to ensure (1) that both groups are optimally managed for HbA1c and comorbidities (dyslipidaemia, HTN, DKD and NAFLD); (2) an equitable baseline comparison for AMT and VSG since health insurance policies commonly require 6 months of continuous medical care prior to their authorisation of MBS; (3) that treatment is consistent across both groups and sites; and to (4) maximise participant retention following the baseline study visit by identifying participants unable to adhere to study visits. Insurance coverage for surgery requires monthly medically supervised visits with either a primary care provider or the bariatric team. As such, the medical group receives monthly contact via telehealth or phone calls from the study team to discuss diabetes education topics such as glycaemic control, nutrition and exercise, to equalise education content and contact time with study staff between the VSG and AMT study arms.

\section{Treatment phase}

Participants completing the run-in period then receive VSG per standard clinical procedures or are designated as continuing to receive AMT. Participants in both groups will continue to be seen every 3 months per ADA guidelines to assess glycaemic control (HbAlc metre download), advance or adjust medications, assess medication adherence, and assess medical and surgical safety outcomes. Both groups will continue to have their T2D and any T2D comorbidities managed by study protocol for a full 2 years following the baseline visit. No attempts to blind study participants nor study staff to study group allocation are made. Figure 1 provides an overview of the 


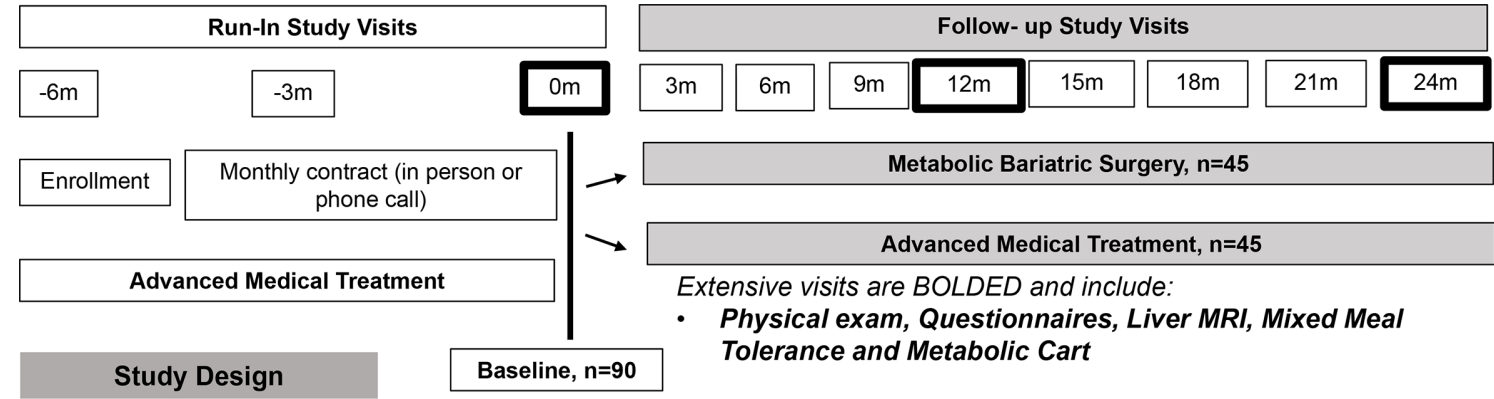

Figure 1 Overview of the Surgical or Medical Treatment for Paediatric Type 2 Diabetes (ST $\left.{ }_{2} \mathrm{OMP}\right)$ study design showing run-in and treatment phases as well as frequencies and timings of study visits.

study design including run-in and treatment phases of the study.

\section{Comprehensive study-outcome visits}

A comprehensive baseline visit occurs prior to the VSG procedure or continuing the AMT intervention. This visit will be repeated at 12 and 24 months. At the comprehensive visit, participants bring in a first morning urine sample and undergo a fasting blood draw and a physical exam that includes vital signs, anthropometrics and Tanner staging. Participants will also complete questionnaires as shown in table 1. A liver MRI examination to quantify liver proton density fat fraction and liver stiffness (magnetic resonance elastography) as a marker of hepatic fibrosis and inflammation, and a mixed meal tolerance test (MMTT) to evaluate beta cell function paired with indirect calorimetry is also conducted. A diet diary, physical activity and sleep monitor are used for the 7 days prior to comprehensive visits to allow adjustment in analyses. Participants will also wear a continuous glucose monitor to assess glycaemic patterns and variability during these 7 days, and in VSG participants, for 2 weeks following their VSG procedure. VSG participants will also have a liver biopsy performed while in the operating room per our institutions' standard clinical practice, and for participants who agree, samples of blood, urine, stool and/ or liver tissue stored in our institutions biobanks. For a list of all procedures, see table 1 . The study radiologist evaluating the liver MRI and the pathologist evaluating the liver biopsy are blinded to study group allocation and clinical data. Following the 24-month visit, participants will return to routine medical care.

\section{Study outcomes}

The $\mathrm{ST}_{2} \mathrm{OMP}$ study primary outcome is the percent of youth achieving HbAlc $<6.0 \%$ at the 12 month posttreatment-allocation visit. An HbA1c of $6 \%$ was chosen because in the TODAY study, this endpoint was attainable with metformin alone in approximately $50 \%$ of adolescents at 1 year and predicted durable glycaemic control. ${ }^{337}$ The HbAlc at 12 months will be measured in a central laboratory at the University of Colorado. The MRI will be interpreted and analysed centrally by a single radiologist study investigator at Cincinnati Children's Hospital and the liver biopsy slides read by a central pathologist at the
National Cancer Institute. Secondary outcomes are listed in table 2 and include remission of comorbidities at 12 and 24 months, and impact of T2D duration and initial HbA1c on the primary outcome. Additional outcomes include measures from the MMTT to assess $\beta$-cell function, $\alpha$-cell function, incretin response, whole-body and adipose insulin sensitivity and carbohydrate and fat oxidation, all which could be mechanisms by which AMT and/ or VSG affect glycaemic control.

\section{Retention}

To aid in study retention, we aligned research visits with timing of required clinical care visits per ADA guidelines for adolescent T2D. Participants are also compensated for their time and travel. Other retention strategies include birthday and holiday cards, as well as small tokens to be given at several of the study visits (examples include back sacks, water bottles and ear buds). Contact information (primary and alternate) is updated at each study visit. Study visit reminders and instructions are provided using a variety of methods including written mailings, phone calls, text messages and e-mail messages.

\section{Sample size}

The target sample size is 90 . The power calculation was based on achieving the main study outcomes in table 1 . For the overall primary endpoint of $\mathrm{HbAlc}<6.0 \%$ and secondary endpoints in (HTN, dyslipidaemia and DKD (using urinary albumin excretion remission)), sample size estimates were calculated using responses to medical treatment in the TODAY clinical trial versus surgical outcomes after RYGB in the Teen-LABS study. ${ }^{24}$ Estimates for the main $\beta$-cell outcome, oral disposition index (insulin secretion * insulin sensitivity), were based on $\beta$-cell responses to medical therapy in the RISE study ${ }^{8}$ and on a non-T2D adolescent cohort who underwent RYGB. ${ }^{8}$ With a proposed sample size of 45 participants per group, the study is powered for the primary and main secondary outcomes, but are also powered if smaller effects are seen with VSG than those observed with RYGB, or as expected, if adolescents with T2D have smaller improvements in $\beta$-cell function, insulin sensitivity and/or insulin secretion in response to MBS compared with the nondiabetic adolescents who comprised the preliminary data. All estimates assume $90 \%$ statistical power, alpha of 0.05 , and 
Table $1 \mathrm{ST}_{2} \mathrm{OMP}$ visit schedule and procedures

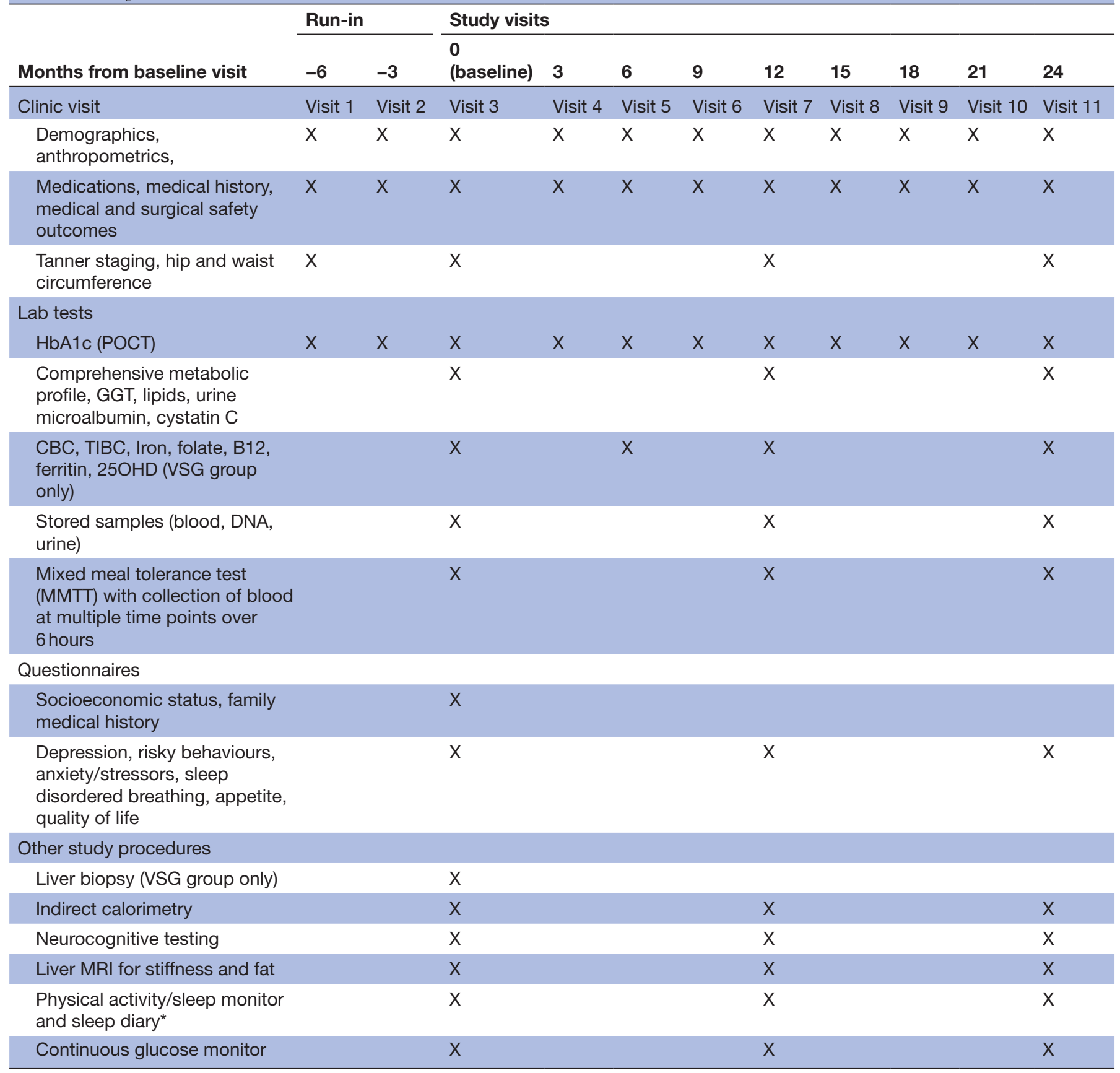

MMTT labs include insulin, pro-insulin, c-peptide, glucagon, free fatty acids, glucagon-like peptide (GLP-1), GIP-1, PYY and ghrelin. ${ }^{*}$ Conducted 3 days prior to study visit.

CBC, complete blood count; GGT, gamma- glutamyl transferase; HbA1c, haemoglobin A1c; POCT, point of care testing; PYY, Peptide YY hormone; TIBC, total iron binding capacity; VSG, vertical sleeve gastrectomy.

$15 \%$ attrition rate. With this sample size, we expect to also have power to explore subgroup analyses by sex and by race.

\section{Statistical analysis}

Following initial data cleaning procedures, descriptive statistics will be calculated to identify any outliers, questionable values, or errors. Frequencies and percentages, mean/median and $\mathrm{SD} / \mathrm{IQR}$ will be examined by treatment group and time point and graphs will be generated. The assumption of normality will be checked. If there is skewness in the data, it will be transformed and analyses will be performed on the transformed variable or we will use non-parametric methods. Change over time and bivariate relationships will be examined by scatter plots, boxplots and contingency tables. For any missing data, concurrent analyses will be performed using complete and available case data or multiple imputation/maximum likelihood techniques. Additionally, 


\begin{tabular}{|c|c|}
\hline Glycaemic control & $\begin{array}{l}\text { Primary: } A 1 c<6.0 \% \text { at } 1 \text { year } \\
\text { A1c }<6.0 \% \text { at } 2 \text { years, time to first } A 1 c \\
<6 \% \text {, number of meds to achieve } A 1 c \\
<6 \% \text {, glycaemic variability }\end{array}$ \\
\hline $\begin{array}{l}\text { Resolution of diabetes } \\
\text { comorbidities } \\
\text { (dyslipidaemia, HTN, } \\
\text { DKD, NAFLD) }\end{array}$ & $\begin{array}{l}\text { LDL-C }<100 \mathrm{mg} / \mathrm{dL} \text { and triglycerides } \\
<150 \mathrm{mg} / \mathrm{dL} \text {, HTN (BP }<95 \text { th percentile), } \\
\mathrm{UAE}<30 \mu \mathrm{g} / \mathrm{mg} \text { and eGFR } \leq 127 \mathrm{~mL} / \\
\mathrm{min} / 1.73 \mathrm{~m}^{2} \text {, hepatic fat }<5 \% \text { by MRI }\end{array}$ \\
\hline$\beta$-Cell function & $\begin{array}{l}\text { Oral disposition index=insulin secretion } \\
\text { (insulinogenic index) * insulin sensitivity } \\
\text { (1/fasting insulin) }\end{array}$ \\
\hline $\begin{array}{l}\text { Adipose insulin } \\
\text { sensitivity }\end{array}$ & Free fatty acid suppression \\
\hline$\alpha$-Cell function & $\begin{array}{l}\text { Fasting glucagon and glucagon area } \\
\text { under the curve }\end{array}$ \\
\hline Incretin response & $\begin{array}{l}\text { Fasting incretins, incretin area under } \\
\text { the curve and hunger scale ratings }\end{array}$ \\
\hline $\begin{array}{l}\text { Carbohydrate and fat } \\
\text { oxidation }\end{array}$ & Fasting and MMTT metabolic cart \\
\hline
\end{tabular}

DKD, diabetic kidney disease; eGFR, estimated glomular filtration rate; HTN, hypertension; LDL-C, low density lipoprotein cholesterol; MMTT, mixed meal tolerance test; NAFLD, nonalcoholic fatty liver disease; UAE, urinary albumin excretion.

pattern-mixture and selection models ${ }^{38}$ will be used for sensitivity analyses.

Analysis of a non-randomised cohort. Treatment selection is often associated with patient characteristics in observational studies. As a result, baseline characteristics of treated patients often differ from those who are untreated. Therefore, propensity score covariate adjustment will be used to account for baseline differences in patient selection of study arms (VSG or AMT). The propensity score allows for analysis of non-randomised data so that it mimics some of the characteristics of a randomised study. Specifically, conditional on the propensity score, the distribution of observed baseline covariates will be similar between VSG and AMT subjects. ${ }^{39}$ Propensity scores will be computed as the predicted probability that a patient selects VSG compared with AMT using logistic regression. Additionally, each of the specific aims will be addressed as described below.

For the primary endpoint, achievement of HbA1c $<6.0 \%$, generalised linear mixed modelling will be used, adjusting for covariates, including, but not limited to: propensity score, treatment group, study visit, age, sex, race/ethnicity, clinical site, baseline $\mathrm{HbAlc}$, duration of T2D and per cent change in BMI from baseline. ORs with 95\% CIs, as well as modelled percentages with $95 \% \mathrm{CIs}$, will be calculated. For secondary outcomes, linear mixed modelling will be used to evaluate the relationship between treatment group and each outcome. Model covariates may include, but are not limited to: propensity score, treatment group, study visit, age, sex, race/ ethnicity, clinical site and per cent change in BMI from baseline.

\section{Reporting adverse events}

Both serious and non-serious adverse events will be reported to the Institutional Review Board. A fourmember independent Data and Safety Monitoring Board consisting of experts in the fields of diabetes and bariatric surgery was appointed to review cumulative data and assess the study progress and conduct, safety data, scientific validity and data integrity, and to provide recommendations to study personnel.

\section{DISCUSSION}

Carefully designed human studies like $\mathrm{ST}_{2} \mathrm{OMP}$ are necessary to advance our understanding of how VSG and AMT affect the pathophysiology underlying youth-onset T2D and to direct the development of new therapeutic strategies to impede the development of complications related to youth-onset T2D. ST 2 OMP will generate an extensive dataset on the outcomes of VSG and AMT. Furthermore, it will also begin a biorepository of blood, urine, stool and liver tissue that will be stored for future use by the primary investigators and will also be available to outside investigators on request, as shown in table 1 . Our over-arching goal is to identify ways to achieve the metabolic benefits of MBS noninvasively, thereby improving the quality and longevity of the lives of adolescents with T2D.

\section{ETHICS AND DISSEMINATION}

The protocol was approved by the Cincinnati Children's Hospital Medical Center and the University of Colorado Institutional Review Boards. Written informed assent and/or consent is sought prior to study enrolment. All participants will receive medical care for T2D and their comorbidities regardless of group enrolment. All study participants will be assigned a study identification number. Only study staff have access to personal information. Study findings will be widely disseminated through peer-reviewed publications and conference presentations.

\section{Author affiliations}

${ }^{1}$ Division of Pediatric Endocrinology, University of Cincinnati and Cincinnati Children's Hospital Medical Center, Cincinnati, Ohio, USA

'Division of Pediatric Surgery, University of Cincinnati and Cincinnati Children's Hospital Medical Center, Cincinnati, Ohio, USA

${ }^{3}$ Division of Pediatric Surgery, University of Colorado and Children's Hospital Colorado, Aurora, Colorado, USA

${ }^{4}$ Division of Pediatric Gastroenterology, Hepatology and Nutrition, University of Cincinnati and Cincinnati Children's Hospital Medical Center, Cincinnati, Ohio, USA

${ }^{5}$ Division of Pediatric Endocrinology, University of Colorado, Children's Hospital Colorado, Aurora, Colorado, USA

${ }^{6}$ Department of Radiology, University of Cincinnati and Cincinnati Children's Hospital Medical Center, Cincinnati, Ohio, USA

${ }^{7}$ Division of Radiology, University of Colorado, Children's Hospital Colorado, Aurora, Colorado, USA

Contributors All authors designed the study and wrote the study protocol. AS and KN wrote the first draft of this manuscript. All authors edited the manuscript and approved the final version for submission.

Funding This work is supported by the National Institutes of Health, Grant Number R01DK119450. This funding source had no role in the design of this study and 
will not have any role during its execution, analyses, interpretation of the data, or decision to submit results.

Competing interests None declared.

Patient and public involvement Patients and/or the public were not involved in the design, or conduct, or reporting, or dissemination plans of this research.

Patient consent for publication Not required.

Provenance and peer review Not commissioned; externally peer reviewed.

Open access This is an open access article distributed in accordance with the Creative Commons Attribution Non Commercial (CC BY-NC 4.0) license, which permits others to distribute, remix, adapt, build upon this work non-commercially, and license their derivative works on different terms, provided the original work is properly cited, appropriate credit is given, any changes made indicated, and the use is non-commercial. See: http://creativecommons.org/licenses/by-nc/4.0/.

ORCID iD

Amy S Shah http://orcid.org/0000-0001-7297-139X

\section{REFERENCES}

1 Hamman RF, Bell RA, Dabelea D, et al. The search for diabetes in youth study: rationale, findings, and future directions. Diabetes Care 2014;37:3336-44.

2 Badaru A, Klingensmith GJ, Dabelea D, et al. Correlates of treatment patterns among youth with type 2 diabetes. Diabetes Care 2014;37:64-72.

3 TODAY Study Group, Zeitler P, Hirst K, et al. A clinical trial to maintain glycemic control in youth with type 2 diabetes. $N$ Engl J Med 2012;366:2247-56.

4 TODAY Study Group. Longitudinal changes in cardiac structure and function from adolescence to young adulthood in participants with type 2 diabetes mellitus: the today follow-up study. Circ Heart Fail 2020;13:e006685.

5 Shah AS, El Ghormli L, Gidding SS, et al. Prevalence of arterial stiffness in adolescents with type 2 diabetes in the today cohort: relationships to glycemic control and other risk factors. J Diabetes Complications 2018;32:740-5.

6 Bjornstad P, Nehus E, El Ghormli L, et al. Insulin sensitivity and diabetic kidney disease in children and adolescents with type 2 diabetes: an observational analysis of data from the TODAY clinical trial. Am J Kidney Dis 2018;71:65-74.

7 RISE Consortium. Metabolic contrasts between youth and adults with impaired glucose tolerance or recently diagnosed type 2 diabetes: II. observations using the oral glucose tolerance test. Diabetes Care 2018;41:1707-16.

8 RISE Consortium, RISE Consortium Investigators. Effects of treatment of impaired glucose tolerance or recently diagnosed type 2 diabetes with metformin alone or in combination with insulin glargine on $\beta$-cell function: comparison of responses in youth and adults. Diabetes 2019;68:1670-80.

9 Tamborlane WV, Barrientos-Pérez M, Fainberg U, et al. Liraglutide in children and adolescents with type 2 diabetes. $N$ Engl J Med 2019;381:637-46.

10 Arslanian S, Bacha F, Grey M, et al. Evaluation and management of Youth-Onset type 2 diabetes: a position statement by the American diabetes association. Diabetes Care 2018;41:2648-68.

11 Zeitler P, Arslanian S, Fu J, et al. ISPAD clinical practice consensus guidelines 2018: type 2 diabetes mellitus in youth. Pediatr Diabetes 2018;19 Suppl 27:28-46.

12 Buchwald H, Estok R, Fahrbach K, et al. Weight and type 2 diabetes after bariatric surgery: systematic review and meta-analysis. Am J Med 2009;122:248-56.

13 Adams TD, Davidson LE, Litwin SE, et al. Weight and metabolic outcomes 12 years after gastric bypass. $N$ Engl J Med 2017;377:1143-55.

14 Aminian A, Brethauer SA, Andalib A, et al. Individualized metabolic surgery score: procedure selection based on diabetes severity. Ann Surg 2017;266:650-7.

15 Amouyal C, Andreelli F. What is the evidence for metabolic surgery for type 2 diabetes? A critical perspective. Diabetes Metab 2017;43:9-17.
16 Rubino F, Nathan DM, Eckel RH, et al. Metabolic surgery in the treatment algorithm for type 2 diabetes: a joint statement by international diabetes organizations. Obes Surg 2017;27:2-21.

17 Schauer PR, Kashyap SR, Wolski K, et al. Bariatric surgery versus intensive medical therapy in obese patients with diabetes. $N$ Engl J Med 2012;366:1567-76.

18 Sjöström L. Review of the key results from the Swedish Obese Subjects (SOS) trial - a prospective controlled intervention study of bariatric surgery. J Intern Med 2013;273:219-34.

19 Sjöström L, Peltonen M, Jacobson P, et al. Association of bariatric surgery with long-term remission of type 2 diabetes and with microvascular and macrovascular complications. JAMA 2014;311:2297-304.

20 Schauer PR, Bhatt DL, Kirwan JP, et al. Bariatric Surgery versus intensive medical therapy for diabetes - 5-year outcomes. $N$ Engl J Med 2017;376:641-51.

21 Schauer PR, Bhatt DL, Kirwan JP, et al. Bariatric surgery versus intensive medical therapy for diabetes--3-year outcomes. $N$ Engl J Med 2014;370:2002-13.

22 RISE Consortium. Metabolic contrasts between youth and adults with impaired glucose tolerance or recently diagnosed type 2 diabetes: I. observations using the hyperglycemic clamp. Diabetes Care 2018;41:1696-706.

23 Inge TH, Courcoulas AP, Jenkins TM, et al. Weight loss and health status 3 years after bariatric surgery in adolescents. $N$ Engl J Med 2016;374:113-23.

24 Inge TH, Laffel LM, Jenkins TM, et al. Comparison of surgical and medical therapy for type 2 diabetes in severely obese adolescents. JAMA Pediatr 2018;172:452-60.

25 Bjornstad P, Hughan K, Kelsey MM, et al. Effect of surgical versus medical therapy on diabetic kidney disease over 5 years in severely obese adolescents with type 2 diabetes. Diabetes Care 2020;43:187-95.

26 Reames BN, Finks JF, Bacal D, et al. Changes in bariatric surgery procedure use in Michigan, 2006-2013. JAMA 2014;312:959-61.

27 Inge TH, Coley RY, Bazzano LA, et al. Comparative effectiveness of bariatric procedures among adolescents: the PCORnet bariatric study. Surg Obes Relat Dis 2018;14:1374-86.

28 de Oliveira VLP, Martins GP, Mottin CC, et al. Predictors of long-term remission and relapse of type 2 diabetes mellitus following gastric bypass in severely obese patients. Obes Surg 2018;28:195-203.

29 Courcoulas AP, Yanovski SZ, Bonds D, et al. Long-Term outcomes of bariatric surgery: a national Institutes of health symposium. JAMA Surg 2014;149:1323-9.

30 Concato J, Shah N, Horwitz RI. Randomized, controlled trials, observational studies, and the hierarchy of research designs. $N$ Engl J Med 2000;342:1887-92.

31 Concato J, Lawler EV, Lew RA, et al. Observational methods in comparative effectiveness research. Am J Med 2010;123:e16-23.

32 Pratt JSA, Browne A, Browne NT, et al. ASMBS pediatric metabolic and bariatric surgery guidelines, 2018. Surg Obes Relat Dis 2018;14:882-901.

33 Armstrong SC, Bolling CF, Michalsky MP, et al. Pediatric metabolic and bariatric surgery: evidence, barriers, and best practices. Pediatrics 2019;144. doi:10.1542/peds.2019-3223. [Epub ahead of print: 27 Oct 2019].

34 Davies MJ, D'Alessio DA, Fradkin J, et al. Management of hyperglycemia in type 2 diabetes, 2018. A consensus report by the American diabetes association (ADA) and the European association for the study of diabetes (EASD). Diabetes Care 2018;41:2669-701.

35 Marso SP, Daniels GH, Brown-Frandsen K, et al. Liraglutide and cardiovascular outcomes in type 2 diabetes. $N$ Engl J Med 2016;375:311-22.

36 Zinman B, Wanner C, Lachin JM, et al. Empagliflozin, cardiovascular outcomes, and mortality in type 2 diabetes. $N$ Engl J Med 2015;373:2117-28.

37 Zeitler P, Hirst K, Copeland KC, et al. Hba1C after a short period of monotherapy with metformin identifies durable glycemic control among adolescents with type 2 diabetes. Diabetes Care 2015;38:2285-92.

38 Verbeke G, Molenberghs G. Linear mixed models for longitudinal data. New York, NY: Springer, 2000.

39 Austin PC. An introduction to propensity score methods for reducing the effects of confounding in observational studies. Multivariate Behav Res 2011;46:399-424. 